\title{
Diagnostic challenge of acute appendicitis: appraisal through modified Alvarado score
}

\begin{abstract}
Acute appendicitis is one of the commonest surgical emergencies. Simple appendicitis can progress to perforation, which is associated with a much higher morbidity and mortality, and surgeons have therefore been inclined to operate when the diagnosis is probable rather than wait until it is certain. Routine history and physical examination still remain most practical diagnostic modalities. Absolute diagnosis of course is only possible at operation and histopathological examination of the specimen. For this reason rate of negative Appendicectomy as reported in the world literature varying from $20-40 \%$. The present study was undertaken in DMC\&H from Jan 2013 to June 2013 to evaluate the usefulness of Modified Alvarado scoring system in reducing the number of negative appendicectomy and to evaluate its sensitivity \& positive predictive value in the diagnosis of acute appendicitis. In this study the negative appendicectomy rate is $10.00 \%$ with the rate being equal in females $(10 \%)$ and males $(10 \%)$
\end{abstract}

Volume 4 Issue 5 - 2017

\author{
Prabhdeep Singh Nain, Anju Bhagtana, \\ Chiranjiv Singh Gill \\ Surgery Professor, Dayanand Medical College \& Hospita
} Ludhiana, India

Correspondence: Prabhdeep Singh Nain, Surgery Professor, Dayanand Medical College \& Hospital Ludhiana, Punjab, India, Email prabhdeepnain@rediffmail.com

Received: November 08, 2016 | Published: July 20, 2017

Keywords: appendicitis, alvarado score, diagnostic

\section{Introduction}

"Diagnosis of appendicitis is usually easy"-thus wrote Sir Zachary Cope, but with the order: "but there are difficulties which need to be discussed". The "difficulty" alluded to by Cope relates to our inability to reliably diagnose appendicitis on clinical grounds. Acute Appendicitis is the most common cause of acute abdomen and of course, a common disease in surgical practice. ${ }^{2}$ It forms $10 \%$ of all emergency abdominal operations. Simple appendicitis can progress to perforation, which is associated with a much higher morbidity and mortality, and surgeons have therefore been inclined to operate when the diagnosis is probable rather than wait until it is certain. ${ }^{3}$ Acute appendicitis is essentially a clinical diagnosis. ${ }^{4}$ Routine history and physical examination still remain the most practical diagnostic modalities. Absolute diagnosis of course is only possible at operation and histopathologic examination of the specimen $^{5}$ over the last two decades different protocols have been introduced and have been tested by different researchers which include Lidverg, Fenyo, Ohman and Alvarado scoring system to make an early diagnosis of this sometimes very elusive disease. Alvarado in 1986 introduced a criterion for the diagnosis of acute appendicitis which was later modified to accommodate additional parameters along with original Alvarado scoring system. ${ }^{6-9}$ The classical Alvarado score included left shift of neutrophil maturation(one score) yielding a total score o10. Kalan et al. ${ }^{10}$ omitted this parameter which is not routinely available in many laboratories, and they produced a modified score. ${ }^{10}$ This study will be carried out to evaluate the Modified Alvarado Scoring System in patients admitted with the diagnosis of Acute Appendicitis. Purpose of different scoring systems for diagnosis of the appendicitis is to facilitate the surgeon and to avert a negative appendicectomy. Modified Alvarado score is a 9point scoring system based on symptoms, clinical signs and leucocyte count as shown in the table given below. The classical Alvarado score includes left shift of neutrophil maturation, which is not routinely done in many laboratories. The modified Alvarado score which includes an extra sign (e.g. cough test, Rovsing sign or rectal tenderness) is helpful in minimizing unnecessary appendectomy and is practical, reliable and easily done. Some studies demonstrated that this extra sign had a sensitivity of $68 \%$ and a specificity of $58 \%$ in the diagnosis of acute appendicitis.

\section{Materials and methods}

A total of 70 patients were admitted in $\mathrm{DMC} \& \mathrm{H}$, out of which 30 patients were included in the study who gave consent. After institutional review board approval was obtained, 30 patients, during the period of 6 months from January 2013 to June 2013 with the provisional diagnosis of Acute Appendicitis admitted in the wards and emergency in the department of surgery in the hospital Dayanand Medical College \& Hospital, Ludhiana were included in the study After collecting the basic clinical data (name, age, gender, history and examination) and collecting the routine lab values), the modified Alvarado score was assigned to each of the patient (Table 1). Diagnosis of acute appendicitis was confirmed by operative findings and histopathological assessment of the appendicectomy specimen. Finally the reliability of Modified Alvarado scoring system was assessed by calculating Negative appendicectomy rate (the proportion of operated patients having normal appendix removed) and Positive predictive value (the proportion of patients with a positive test result who actually have the disease)

Table I Modified alvarado scoring system

\begin{tabular}{ll}
\hline Modified alvarado score & Score \\
\hline Migratory right iliac fossa pain & 1 \\
Anorexia & 1 \\
Nausea/vomiting & 1 \\
Signs & \\
Tenderness right lower quadrant & 2 \\
Rebound tenderness right iliac fossa & 1 \\
Pyrexia >=37.5 & 1 \\
Extra sign(cough test, rovsing sign or rectal tenderness) & \\
Investigations & 1 \\
Leukocytosis & 2 \\
\hline
\end{tabular}




\section{Results}

In this series of 30 cases, the patients who presented with the acute symptoms and pre-operatively diagnosed to have acute appendicitis were studied. Out of the total 30 cases that were studied and operated based on the clinical scoring system. Out of the 30 cases that were studied, 27 had acutely inflamed appendix. The percentage of the inflamed appendix found on operation was $90 \%$ and $10 \%$ of the patients were found to have normal appendix i.e, 3 out of the 30patients were found to have normal appendix. In this study, the males accounted for $66.7 \%$ and the females accounted for $33.3 \%$. Out of the 30patients, total number of males was 20 and the total number of females was 10. The number of patients was highest in the age group 21-30years (40\%) followed by $9-20 y e a r s ~(26.7 \%)$. The least were in the age group 45years and above. Out of 30patients, 20 were male $(66.6 \%)$ and 10 were female (33.3\%). Most of the patients were of younger age group. This result shows that there is predominance in the younger age group and incidence peaks around 15 to 30 years and decreases as the age progresses. Migratory RIF pain was in 28patients out of the 30patients i.e. $93.3 \%$ of the patients. Anorexia was symptom in $90 \%$ of the patients i.e. in 27 cases it was present. Nausea and vomiting was present in $83.3 \%$ of the cases. Tenderness RLQ was present in $93.3 \%$ of the cases. Rebound tenderness was present in $70 \%$ of the patients. Raised temperature was found in $53.3 \%$ of the patients, leukocytosis in $80 \%$ of the patients. Rovsing's was present only in $40 \%$ of the patients (Table 2).

Table 2 Presentation of clinical features

\begin{tabular}{lll}
\hline Clinical features & No. & Percentage (\%) \\
\hline Migratory RIF Pain & 28 & 93.3 \\
Anorexia & 27 & 90 \\
Nausea \&Vomiting & 25 & 83.3 \\
Tenderness RLQ & 28 & 93.3 \\
Rebound Tenderness RLQ & 21 & 70 \\
Elevated Temperature & 16 & 53.3 \\
Leukocytosis & 24 & 80 \\
Extra Signs (Rovsing's Sign) & 12 & 40 \\
\hline
\end{tabular}

Total of 30patients were operated, out of which 30 were male and 10 were female. 15 male patients having score $>7$ had acute appendicitis, 1patient had normal appendix. Male patients having score of 5-6 were 4, out of which 3 patients had acute appendicitis, 1 patient had normal appendix. In 9 female patients having a score 7-10, 9 had acute appendicitis, no one having normal appendix. In 1 female with score 5-6, 0 had normal appendix. Acute Catarrhal Appendicitis was present in $73.3 \%$ of the cases. Acute Gangrenous Appendicitis was present in $10 \%$ of the cases. Acute perforated appendicitis was present in $6.7 \%$ of the cases. The most common of the all was Acute Catarrhal Appendicitis. Out of the 30patients, patients having score $>7$ were 16 males and 9 female patients. Of these 15 male patients had acute appendicitis and 9 female patients had acute appendicitis. On histopathological examination, 1 patient having normal appendix with MAS $>7$ Out of the 30patients, patients having score 5-6 were 4males and 1 female patients. Of these 3 male patients had acute appendicitis and 1 male patient had acute appendicitis. On histopathological examination, 1patient having normal appendix with MAS5-6. Increased proportion $(40 \%)$ of negative appendicectomy is noticed for the Alvarado Score 5-6 and significantly decreased proportion $(10 \%)$ negative appendicectomy is noticed for the Alvarado Score $7-10$ with an Odds ratio of 4.98 and $\mathrm{P}$ value 0.014 (Table 3 ). The positive predictive value in patients having score $>7$ was $93.75 \%$ in case of males and $100 \%$ in case of females. Similarly the positive predictive value in patients having score $5-6$ was $75 \%$ in case of males and $0 \%$ in case of the females (Table 4 ). In this study the negative appendicectomy rate is $10.00 \%$ with the rate being equal in females $(10 \%)$ and males (10\%) (Table 5).

Table 3 Diagnostic value of modified alvarado score.

\begin{tabular}{llllllll}
\hline \multirow{2}{*}{ HPE } & \multicolumn{3}{l}{ MAS } & \multicolumn{5}{l}{ Total } & P \\
\cline { 2 - 6 } & \multicolumn{2}{l}{ MAS (5-6) } & \multicolumn{2}{l}{ MAS (7-9) } & \multirow{2}{*}{ No. } & \%Age & Value \\
\cline { 2 - 6 } & No. & \%Age & No. & \%Age & & \\
Appendicitis & 3 & 60 & 24 & 96 & 27 & 90 & \\
Normal & 2 & 40 & 1 & 4 & 3 & 10 & 0.014 \\
Total & 5 & 100 & 25 & 100 & 30 & 100 & \\
\hline
\end{tabular}

Table 4 Final results of modified alvarado score

\begin{tabular}{lllll}
\hline $\begin{array}{l}\text { Total no. of } \\
\text { patients }\end{array}$ & Score $>\mathbf{7}$ & Appendicitis & $\begin{array}{l}\text { Positive predictive } \\
\text { value }\end{array}$ \\
\hline Male & 20 & 16 & 15 & 93.75 \\
Female & 10 & 9 & 9 & 100 \\
Score 5-6 & & & & \\
Male & 20 & 4 & 3 & $75 \%$ \\
Female & 10 & 1 & 0 & $0 \%$ \\
\hline
\end{tabular}

Table 5 Negative appendicectomy rates

\begin{tabular}{llll}
\hline \multirow{2}{*}{ Sex } & \multirow{2}{*}{ No. } & \multicolumn{2}{l}{ Total } \\
\cline { 3 - 4 } Female & 10 & 1 & No. \\
Male & 20 & 2 & $10.00 \%$ \\
Total & 30 & 3 & $10.00 \%$ \\
\hline
\end{tabular}

\section{Discussion}

Throughout the world acute appendicitis remains a common abdominal emergency. Early and accurate diagnosis of acute appendicitis is essential so as to reduce the morbidity and mortality associated with delayed diagnosis and its complications. In addition to significant morbidity and mortality, negative appendicectomy is also responsible for loss of precious staff hours and financial resources. Though there are lots of advances in the diagnostic field with the invention of sophisticated investigations, diagnosis of acute appendicitis remains an enigma for the attendant surgeon. None of the investigations like ultrasound, Computed tomography scan conclusively diagnose appendicitis. A thorough clinical examination with basic investigations like TLC count remains the cornerstone in the diagnosis of acute appendicitis. A number of clinical scoring systems have been used as complimentary aid in diagnosis of acute appendicitis. Initial assessment can be improved by use of a clinical 
scoring system. The present study was undertaken to evaluate the usefulness of Modified Alvarado scoring system in reducing the number of negative appendicectomy and to evaluate its sensitivity \& positive predictive value in the diagnosis of acute appendicitis. Our results and observations were discussed and compared with various other studies. In this study, the males accounted for $66.7 \%$ and the females accounted for $33.3 \%$. The number of patients were highest in the age group 21 to 30 years $(40 \%)$ followed by 9 to 20 years $(26.7 \%)$. The least were in the age group 40years and above (10\%). Out of 30 patients, $20(66.6 \%)$ were males and $10(33.3 \%)$ were females. This showed that there was predominance in the males as compared to the females. Most of the patients were of the younger age group. This result shows that there is predominance in the younger age group and the incidence peaks around 21 to 30years and decreases as the age progresses. Pain was the commonest presenting symptom and migratory right iliac fossa pain was observed in $93.3 \%$ of all the cases of the present series. No significant difference in the duration of pain existed between acute appendicitis and other pathological conditions like renal/ureteric colic and gynaecological disorders in the study. Other symptoms observed were anorexia in $90 \%$ of the cases and nausea/vomiting in $83.3 \%$ of the cases. Majority of patients presented within 48 hours of the onset of the pain, with most of them presenting within 12 to 24 hours of the onset of the pain.

On clinical examination tenderness at McBurney's was the commonest sign (93.3\%). Rebound tenderness was present in the $70 \%$ of the cases. In these cases, there was presence of local peritonitis or when inflamed appendix was more interiorly placed. Rovsing's sign was positive in $40 \%$ of the cases and obturator test was positive only in $6.7 \%$ of the cases. On laboratory tests, leukocytosis was seen in $80 \%$ of the cases. These results were in confirmation with the study done by Lewis et al. Out of the 20males, the score of more than 7 were 16 and score of 5-6 were 4 males. Out of the 10 female patients, 9 had a MAS of more than 7 and 1 was in the group of 5-6. Total of 30 patients were operated, out of which 30 were male and 10 were female. 15 patients having score $>7$ had acute appendicitis, 1 patient had normal appendix. Male patients having score of 5-6 were 4, out of which 3 patients had acute appendicitis, 1 patient had normal appendix. In 9female patients having a score 7-10,9 had acute appendicitis, no one having normal appendix. In 1 female with score 5-6, 3 had normal appendix. In our series when the score was more than 7 indicating strong possibility of intra-abdominal infection localized to the Right Iliac fossa, emergency surgery was performed within 6hours. These patients had badly inflamed appendix with impending perforation once again indicating the sensitivity and specificity of the scoring system. In patients in whom score was between 5 and 6 were observed for a period of 12-24hours and re-assessed, where there was persistence of abdominal tenderness with increased WBC count appendicectomy was carried out. These patients were also found to have congested and inflamed appendix. In this study the sensitivity, specificity and positive predictive value were $100 \%, 0 \%$ and $91 \%$ respectively. The positive predictive value shown by this study is comparable with the studies done by M Kalan, KA Malik and TD Owen who reported $87.5 \%, 85.3 \%$ and $87.4 \%$ respectively. This study also revealed that Modified Alvarado scoring system is more helpful in lowering negative appendicectomy rates. Overall the negative appendicectomy rate was equal in males and females. Since intraabdominal infection in females, particularly lower abdomen can be quite confusing, it is difficult to differentiate acute appendicitis from gynaecological conditions like twisted ovarian cyst and PID, laparoscopy and abdomino-pelvic Ultrasonography scan can be advised as a diagnostic tool to minimize negative appendicectomy. Increased proportion of negative appendicectomy is noticed for the modified Alvarado Score 5-6 and significantly decreased proportion negative appendicectomy is noticed for the modified Alvarado Score 7-10.

A similar study was conducted on one hundred twenty eight patients admitted to Al-Kind teaching hospital from June 2009 to June 2010 by Raid E Rasam ${ }^{11}$ study aimed to assess the reliability and the reproducibility of this score for patients presenting in the emergency room with acute right lower quadrant abdominal pain. ${ }^{11}$ A prospective manometric study concluded that the Alvarado score is a reliable, cheap and reproducible tool for the diagnosis of acute appendicitis in the emergency room. ${ }^{12}$ In our present study, usefulness of the modified Alvarado scoring system was demonstrated beyond doubt by reducing number of negative laparotomy especially in men. However in women the negative laparotomy was high and this can be avoided by using laparoscopy and abdomino-pelvic Ultrasonography scan as a diagnostic tool.

\section{Acknowledgements}

None.

\section{Conflict of interest}

The author declares no conflict of interest.

\section{References}

1. Wagner JM, McKinney WP, Carpenter JL. Does this patient have appendicitis? JAMA. 1996;276(19):1589-1594.

2. Schwartz SI, Shires GT, Spencer FC. Principles of Surgery. 6th ed. New York: USA: McGraw-Hill; 1994. p. 1307-1318.

3. Hoffmann J, Rasmussen O. Aids in the diagnosis of acute appendicitis. $\mathrm{Br}$ J Surg. 1989;76(8):774-790.

4. Williams NS, Bulstrode CJK, O'Connell PR. The vermiform appendix Bailey \& Love's Short practice of Surgery 25thed. 2008;67:1204-1218.

5. Dey S, Mohanta PK, Baruah AK, et al. Alvarado scoring in acute appendicitis-a clinicopathological correlation. Indian $J$ Surg. 2010;72(4):290-293.

6. Ohmann C, Yang Q, Franke C. The abdominal pain study group. Diagnostic scores for acute appendicitis. Eur J Surg. 1995;161(4):273-281.

7. Owen TD, Williams H, Stiff G, et al. Evaluation of the Alvarado score in acute appendicitis. J Soc Med. 1992;85(2):87-88.

8. Alvarado A. A practical score for the early diagnosis of acute appendicitis. Ann Emerg Med. 1986;15(5):557-564.

9. Macklin CP, Radcliffe GS, Merei JM, et al. A prospective evaluation of modified Alvarado score for acute appendicitis in children. Ann R Coll Surg Engl. 1997;79(3):203-205.

10. Kalan M, Rich AJ, Talbot D, et al. Evaluation of the modified Alvarado score in the diagnosis of acute appendicitis: a prospective study. Ann $R$ Coll Surg Eng. 1994;77(2):157.

11. Raid E, Rassam Al. Modified Alvarado Scoring System- How Much Helpful? Kindy Col Med J. 2011;7(2):148-156.

12. Pouget-Baudry Y, Mucci S, Eyssartier E, et al. The use of the Alvarado score in the management of right lower quadrant abdominal pain in the adult. J Visc Surg. 2010;147(2):40-44. 